\title{
A NOVEL PREDICATE FOR ACTIVE REGION MERGING IN AUTOMATIC IMAGE SEGMENTATION
}

\author{
S.Muthamizhselvi ${ }^{1}$, D.Jeyakumari ${ }^{2}$, R.Kannan $^{3}$ \\ ${ }^{I}$ PG Scholar, ${ }^{2}$ Associate Professor, ${ }^{3}$ Assistant Professor, \\ ${ }^{1,2,3}$ Department of ECE, RVS college of Engineering and Technology, Coimbatore, Tamilnadu, India \\ muthamizhselvi24@gmail.com,dgjeyakumari@gmail.com,kannanvlsi@gmail.com
}

\begin{abstract}
Image segmentation is an elementary task in computer vision and image processing. This paper deals with the automatic image segmentation in a region merging method. Two essential problems in a region merging algorithm: order of merging and the stopping criterion. These two problems are solved by a novel predicate which is described by the sequential probability ratio test and the minimal cost criterion. In this paper we propose an Active Region merging algorithm which utilizes the information acquired from perceiving edges in color images in $L^{*} a^{*} b^{*}$ color space. By means of color gradient recognition method, pixels with no edges are clustered and considered alone to recognize some preliminary portion of the input image. The color information along with a region growth map consisting of completely grown regions are used to perform an Active region merging method to combine regions with similar characteristics. Experiments on real natural images are performed to demonstrate the performance of the proposed Active region merging method.
\end{abstract}

Index Terms: Adaptive threshold generation, CIE $L^{*} a^{*} b^{*}$ color gradient, region merging, Sequential Probability Ratio Test (SPRT).

\section{INTRODUCTION:}

For some applications such as image recognition (or) image compression, we cannot process the whole image directly for the reason as it is inefficient and impractical. Therefore we are going for image segmentation before recognition (or) compression. Image segmentation is a basic yet still a demanding problem in computer vision and image processing. Image segmentation is a key process in object recognition, target tracking, content-based image retrieval, and medical image processing, video, and computer vision applications like medical image analysis such as locating tumors and other pathologies, in locating objects in satellite images, in face recognition, fingerprint recognition etc. The goal of image segmentation is to partition an image into a certain number of pieces that have coherent features (color, texture, etc.) and, in the meanwhile, to group the meaningful pieces together for the convenience of perceiving. In many practical applications, as a large number of images are needed to be handled, human interactions involved in the segmentation process should be as less as possible. For this several general-purpose algorithms and techniques have been developed for image segmentation.

There is a large amount of literature on automatic image segmentation. For Example, the spatiotemporal segmentation is to identify the objects present in the scene represented in a video sequence. This technique processes two consecutive frames at a time. Regions are merged based on their mutual spatiotemporal similarity. Modified Kolmogorov -Smirnov test for estimating the temporal similarity. This test efficiently uses temporal information in both the residual distribution and the motion parametric representation. There are two complementary graph-based clustering rules are proposed, namely, the strong rule and the weak rule. The weak rule is applied after the strong rule. It cannot combine the tracking information into the segmentation process. The objects forming the scene are not tracked through time [8]. A hybrid multidimensional image segmentation algorithm, which combines edge and region-based techniques through the morphological algorithm of watersheds. An edge-preserving statistical noise reduction approach is used as a preprocessing stage in order to compute an accurate estimate of the image gradient. Then, an initial partitioning of the image into primitive regions is produced by applying the watershed transform on the image gradient magnitude. This initial segmentation is the input to a computationally efficient hierarchical region merging process that produces the final segmentation. The latter process uses the region adjacency graph (RAG) and nearest neighbor graph (NNG) representation of the image regions. The final segmentation provides, due to the RAG, one-pixel wide, closed, and accurately localized contours/surfaces. It does not involve in the segmentation of moving 3D images [13].

Different algorithms, based on a combination of two structures of graph and of two color image processing methods, in order 
to segment color images. The structures used in this study are the region adjacency graph and the line graph associated. It shows how these structures can enhance segmentation processes such as region growing or watershed transformation. The principal advantage of these structures is that they give more weight to adjacency relationships between regions than usual methods [1]. Many tasks in computer vision involve assigning a label (such as disparity) to every pixel. A common constraint is that the labels should vary smoothly almost everywhere while preserving sharp discontinuities that may exist, e.g., at object boundaries. These tasks are naturally stated in terms of energy minimization. In this paper, we consider a wide class of energies with various smoothness constraints. Global minimization of these energy functions is NP-hard even in the simplest discontinuity-preserving case. Two algorithms based on graph cuts that efficiently find a local minimum with respect to two types of large moves, namely expansion moves and swap moves. These moves can simultaneously change the labels of arbitrarily large sets of pixels. It can find only a local minimum [2].

A database containing 'ground truth' segmentations produced by humans for images of a wide variety of natural scenes. They define an error measure which quantifies the consistency between segmentations of differing granularities and find that different human segmentations of the same image are highly consistent. Use of this dataset is demonstrated in two applications: (1) evaluating the performance of segmentation algorithms and (2) measuring probability distributions associated with Gestalt grouping factors as well as statistics of image region properties [6]. The technique of scale multiplication is analyzed in the framework of canny edge detection. A scale multiplication function is defined as the product of the responses of the detection filter at two scales. Edge maps are constructed as the local maxima by thresholding the scale multiplication results. The detection and localization criteria of the scale multiplication are derived. At a small loss in the detection criterion, the localization criterion can be much improved by scale multiplication. The product of the two criteria for scale multiplication is greater than that for a single scale, which leads to better edge detection performance. [13]

A current level set image segmentation methods, the number of regions assumed to be known beforehand. As a result, it remains constant during the optimization of the objective functional. This study investigates a region merging prior related to regions area to allow the number of regions to vary automatically during curve evolution, thereby optimizing the objective functional implicitly with respect to the number of regions. The paper gives a statistical interpretation to the coefficient of this prior to balance its effect systematically against the other functional terms. They demonstrate the validity and efficiency of the method by testing on real images of intensity, color, and motion [4]. The GSEG algorithm is primarily based on edge detection, dynamic region growth and multi-resolution region merging [19].

\section{PROPOSED METHOD:}

The proposed algorithm consists of three steps. First, an edge detection algorithm is used to produce an edge-map used in the creation of adaptive gradient thresholds, which selects the regions of nearby pixels that display similar gradient and color values, producing an initial segmentation map. Second, a region growing algorithm is used for growing the regions. Finally, an active region merging algorithm is used for merging the similar regions by using a novel predicate $\mathrm{P}$.

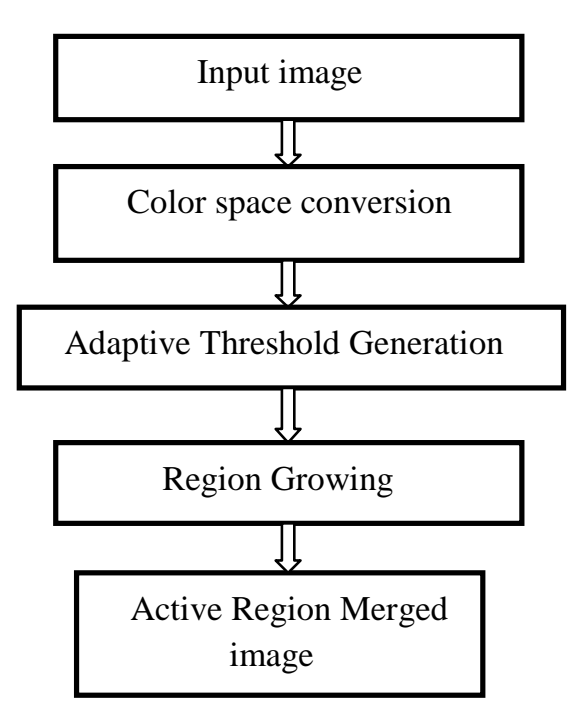

Fig -1: Block diagram of the proposed algorithm

\subsection{Edge Detection:}

The perceived areas with no edges inside them are the preliminary clusters or seeds selected to initiate the segmentation of the image. Assuming that an image is a function $\mathrm{f}(\mathrm{x}, \mathrm{y})$, the gradient can be defined as its initial derivative $\nabla f=[(\partial f / \partial x) ;(\partial f / \partial y)]$. For a vector field $\mathrm{f}$, the gradient vector to be,

$$
D(x)=\left[\begin{array}{cc}
D_{1} f_{1}(x) \ldots \ldots \ldots \ldots \ldots . . & D_{n} f_{1}(x) \\
D_{1} f_{m}(x) \ldots \ldots \ldots \ldots \ldots . . . & D_{n} f_{m}(x)
\end{array}\right]
$$

where Djfk is the first partial derivative of the kth component of $f$ with respect to the jth component of $x$. For a threechannel color image, the gradient can be calculated as: let $\mathrm{a}, \mathrm{b}, \mathrm{c}$ denotes each color channel and $\mathrm{x}, \mathrm{y}$ denote the spatial coordinates for a pixel. It is expressed as: 


$$
\begin{aligned}
& u=\left(\frac{d a}{d x}\right)^{2}+\left(\frac{d b}{d x}\right)^{2}+\left(\frac{d c}{d x}\right)^{2} \\
& v=\left(\frac{d a}{d x} \frac{d a}{d y}\right)+\left(\frac{d b}{d x} \frac{d b}{d y}\right)+\left(\frac{d c}{d x} \frac{d c}{d y}\right) \\
& w=\left(\frac{d a}{d y}\right)^{2}+\left(\frac{d b}{d y}\right)^{2}+\left(\frac{d c}{d y}\right)^{2}
\end{aligned}
$$

\subsection{Adaptive Threshold Generation:}

The Active Region Merging algorithm is started with a color space conversion of the input image from RGB to CIE $L^{*} A * B$. The magnitude of the gradient $G(i, j)$ of the color image can be calculated by using $L^{*} A^{*} B$. Primarily, the aim is to choose a threshold for the initiation of the seed generation procedure. Preferably, a threshold value could be choosing to offer the most edges, where as ignoring the noise in images. A single threshold that may accurately define the boundary of a given region can permit the other regions to merge wrongly. So, we are choosing two initial threshold values for the low and high gradient content in the image. The high initial threshold can be used for images which have a large gradient values over a narrow range and a low initial threshold value can be used for images which have a large gradient values over a wide range. It guarantees that all significant low gradient regions are obtained as initial seeds. Low initial threshold is denoted as $\lambda$ and high initial threshold is denoted as $\lambda+5$.

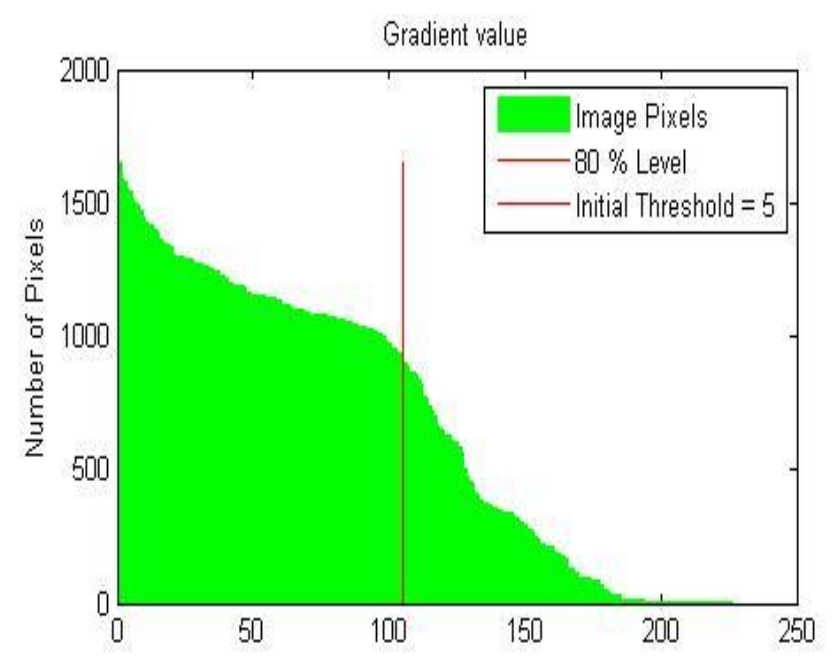

Fig -2: Adaptive Threshold Generation

\subsection{Seed Generation:}

Preliminary seeds are generated by perceiving all the regions in the image whose gradient values fall below the initial thresholds $\lambda$, and $\lambda+5$. If no regions present under this threshold, the edge value is increased until regions are identified. The Preliminary Seed Generation makes use of individual size necessity to select the preliminary seeds, so as to prevent numerous seed generation within identical and associated regions. The initial condition is to implement that seeds to be superior to $0.5 \%$ of the image when looking for regions with a threshold value lower than $\lambda$.The basis of this rule is that frequently backgrounds have false edges produced by illumination or other varying factors in this gradient range. The second condition is to implement that seeds to be superior to $0.25 \%$ of the image in the range $\lambda$ to $\lambda+5$, since it is necessary to differentiate the regions. For distinguishing purposes the pixels creating each seed obtain an exclusive label, the mutual set of labeled seeds is called as the Parent Seeds (PS).

\subsection{Region Growing:}

The Parent Seeds (PS) grows by increasing the threshold a single component at a time, for example from 20 to 21. After each growth, recognition of new regions or child seeds that falls below the new threshold happens. These child seeds requires to be categorized in to adjacent-to-existent or non adjacent seeds. The non adjacent seeds are surplus, because they can only be added at the starting of an each adaptive threshold stage. In order to make the region growth method capable, it is necessary to recognize the parent seed to which the child is adjacent. The goal is to process all adjacent child seeds in a vectorized method. To attain this task, we go on to perceive the outside edges of the map using a nonlinear spatial filter. The filter is defined as follows:

$$
F(i, j)=\left\{\begin{array}{l}
0, \text { if } P S(i, j)>0 \\
0, \text { if } \sum_{i, j \in \beta} P S(i, j)=0 \\
1, \text { Otherwise }
\end{array}\right.
$$

where $\beta$ is the neighborhood. The result of pertaining this filter creates the boundary of the PS map. Assign the label for each pixel in the image. Finally, it produces the region growth map.

\subsection{Region Merging:}

Region merging is the process of merging the two similar regions. Similarity is based on color, texture, grey level etc. Region merging operations eliminates false boundaries and spurious regions by merging adjacent regions that belongs to the same object. It controls the over segmentation problem. Here, the Active Region Merging process occurs by using a region merging predicate $\mathrm{P}$ and cue consistency.

\subsubsection{Region Merging Predicate:}

The proposed predicate is based on measuring the similarity between the pixels along the boundary of two regions. The similarity between the two regions $\mathrm{R} 1$ and $\mathrm{R} 2$ can be calculated by using an equation given below: 


$$
S\left(\boldsymbol{R}_{1}, \boldsymbol{R}_{2}\right)=\min \left(w\left(n_{i}, n_{j}\right)\right)
$$

where ni and $\mathrm{nj}$ are the set of nodes corresponding to the regions, ni belongs to $\mathrm{R} 1$ and $\mathrm{nj}$ belongs to $\mathrm{R} 2$ and (ni,nj) belongs to edge E. An edge has a corresponding weight $\mathrm{w}$. We obtain the similarity between the two neighboring regions by using a minimal weight edge connecting them. The merging predicate $\mathrm{P}$ will decide whether there is an indication of merging between the two regions. It requires two features: a similarity measure, which is used to find out the candidate region for merging and the consistency property, which verifies if the regions are homogenous. Merge R1 and R2 if and only if they are the most similar neighbors.

$$
P\left(R_{1}, R_{2}\right)=\left\{\begin{array}{c}
\text { true if }(a) S\left(R_{1}, R_{2}\right)=\min S\left(R_{1}, R_{a}\right)=\min S\left(R_{2}, R_{b}\right) ; \text { and } \\
\quad(b) R_{1} \text { and } R_{2} \text { are consistent. } \\
\text { false otherwise }
\end{array}\right.
$$

where $\mathrm{Ra}$ and $\mathrm{Rb}$ is the neighboring region of $\mathrm{R} 1$ and $\mathrm{R} 2$. Condition (a) indicates that the connecting edge between R1 and R2 to be the minimal one in either of the neighborhood. Condition (b) acts as a stopping criterion.

\subsubsection{Cue Consistency:}

In order to attain the similar regions in region merging, the proposed predicate $\mathrm{P}$ verifies the consistency of the regions. The region information is obtained by the cues. The selection of cues can be intensity, color, texture and so on. The spreading of cues depends on the consistency of the pair of neighboring regions. In this paper, we are going to use a sequential probability ratio test method. Assuming that parameter $\phi$ is connected to the dissemination of random cues $y$. There are two premises involved here: a couple of regions is "consistent" and "inconsistent". The consistent premises is denoted as H0 : $\phi=\phi 0$ and inconsistent premises is denoted as H1 : $\phi=\phi 1$. The application of the SPRT to the consistency test of cues is expressed as follows. The sequence of following likelihood ratio $\delta \mathrm{j}$ is

$$
\delta_{j}=\log \frac{P_{0}\left(y_{j} / \phi_{0}\right)}{P_{1}\left(y_{j} / \phi_{1}\right)}, j=1,2 \ldots \ldots \ldots n
$$

where $\mathrm{P} 0(\mathrm{yj} / \phi 0)$ and $\mathrm{P} 1(\mathrm{yj} / \phi 1)$ are the disseminations of cues. We are going to use the Gaussian distribution model to fairly accurate the cue distributions. The two conditional probabilities are expressed as:

$$
\begin{aligned}
& \left\{P_{0}\left(y / \phi_{0}\right)=\lambda_{1} \exp \left(-\left(K_{b}-K_{a+b}\right)^{T} X_{K}^{-1}\left(K_{b}-K_{a+b}\right)\right)\right. \\
& \left\{P_{1}\left(y / \phi_{1}\right)=1-\lambda_{2} \exp \left(-\left(K_{b}-K_{a}\right)^{T} X_{K}^{-1}\left(K_{b}-K_{a}\right)\right)\right.
\end{aligned}
$$

where $\mathrm{Ka}$ and $\mathrm{Kb}$ are the standard color of sampled data in regions $\mathrm{a}$ and $\mathrm{b}$, and $\mathrm{Ka}+\mathrm{b}$ is the standard value of the samples union. $\mathrm{Xk}$ is the covariance matrix of the regions and $\lambda 1$ and $\lambda 2$ are the scalar parameters. The arrangement of likelihood ratio is the addition of individual $\delta$ j, i.e.,

$$
\delta=\sum_{j=1}^{N} \delta_{j}
$$

where $\mathrm{N}$ is the first integer. By using SPRT method, we can execute the test with a realistic estimation. It can be expressed as $C=\log (1-\beta) / \alpha$ and $D=\log \beta(1-\alpha)$, where $\alpha$ and $\beta$ are the probabilities of decision error given by,

$\alpha=\mathrm{Pt}\{$ Rejecting $\mathrm{H} 1$ when $\mathrm{H} 1$ is true $\}$

$\beta=\mathrm{Pt}\{$ Accepting $\mathrm{H} 1$ when $\mathrm{H} 0$ is true $\}$

The selection of $\alpha$ and $\beta$ gives the region merging feature. $\alpha$ and $\beta$ are set to a permanent value of 0.05 .

\subsubsection{Active Region Merging Algorithm (ARM):}

The proposed ARM method deals with the region merging predicate P. By taking the region merging as an important problem, the aim is to assign each region a label, such that the region that belongs to the same object will have the same label. There are two types of labels for regions: initial label and final label. The test of consistency and inconsistency is based on the error probabilities of cue decisions $\alpha$ and $\beta$. The error probabilities are used for finding the homogenous regions. If any non homogenous regions found, the more number of tests are used for the decisions. The regions are arranged in a sequential order from initial label to final label. It depends on the minimum weight between the two regions. The whole image is the union of all the regions, $\mathrm{F}$ is the sum of transition costs of overall regions.

$$
F=\sum_{R_{j}} F_{j}
$$

where $\mathrm{Fj}$ is the addition of transition costs of $\mathrm{Rj}$.

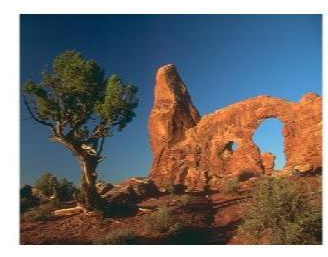

(a)

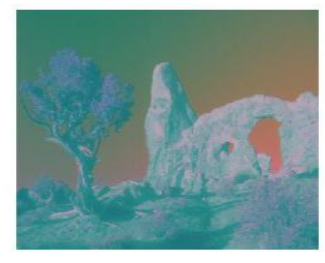

(b) 


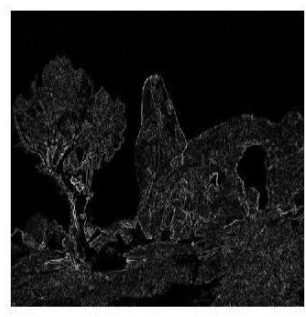

(c)

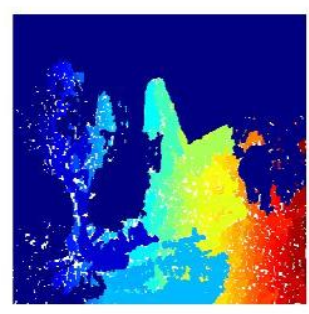

(d)

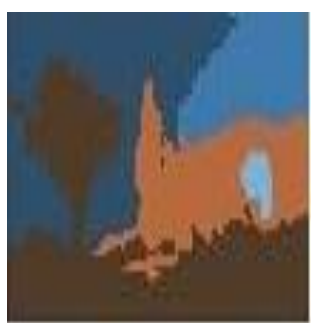

(e)

Fig-3 (a) Input image (b) Color space conversion (c) Gradient image (d) Region Growing (e) Region Merging.

\section{RESULTS AND DISCUSSIONS:}

\subsection{Choices for Parameters:}

In the proposed ARM method, there are five parameters. They are $\mathrm{q}, \alpha, \beta, \lambda 1, \lambda 2$ that controls the consistency premises evaluation.

Here, $\mathrm{q}$ is used to select the amount of data for the random test. $\alpha$ and $\beta$ are used for the probability of accepting the consistency and inconsistency and for rejecting the consistency and inconsistency. In our method, $\alpha$ and $\beta$ are set to a constant value of 0.05 and $\lambda 1$ ranges from 0.01 to 0.05 and $\lambda 2=1$.

\subsection{Performance Evaluation:}

In our method, we are calculating the accuracy for region growing and Active region merging. The Accuracy can be expressed as

$$
\text { - Accuracy }(\%)=\frac{\mathrm{TP}+\mathrm{TN}}{\mathrm{N}} \times 100 \%
$$

The terms used to measure the test performance are true positive (TP), true negative (TN) and total number of images (N). Results obtained for each test images are given in Table I.

\subsection{Comparison of proposed method with GSEG algorithm:}

Here we are going to compare the segmentation results of ARM with GSEG algorithm. GSEG performs the segmentation process by using texture model, color edge detection and cluster formations. There are two parameters used here. They are texture channel bandwidth and spatial bandwidth. The segmented image is based on the texture. The proposed method contains five parameters $\mathrm{q}, \alpha, \beta, \lambda 1, \lambda 2$. Compared to GSEG algorithm, the proposed method produces good localization of boundaries. It reduces the misclassified regions and other irregularities of the gradient.

Table -1: Region Merging Accuracy by GSEG and ARM for various images

\begin{tabular}{|l|c|l|c|}
\hline Image name & $\begin{array}{l}\text { Region } \\
\text { Growing } \\
\text { Accuracy } \\
(\%)\end{array}$ & $\begin{array}{l}\text { Region } \\
\text { Merging } \\
\text { Accuracy by } \\
\text { GSEG }(\%)\end{array}$ & $\begin{array}{l}\text { Region } \\
\text { Merging } \\
\text { Accuracy by } \\
\text { ARM }(\%)\end{array}$ \\
\hline Tree & 85 & 87 & 89 \\
\hline Church & 93 & 95 & 98 \\
\hline Flowers & 82 & 84 & 88 \\
\hline Man & 80 & 83 & 85 \\
\hline
\end{tabular}

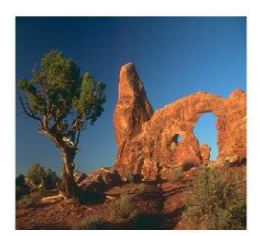

(a)

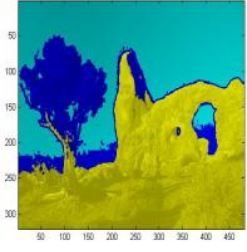

(b)

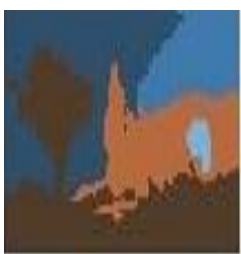

(c)
Fig-4 (a) Input image (b) GSEG image (c) ARM image

\section{CONCLUSIONS}

In this paper, we proposed a novel predicate for merging the regions in automatic image segmentation. The Predicate $\mathrm{P}$ is used to determine the evidence of merging between the two neighboring regions. The Predicate is defined by using Sequential Probability Ratio Test (SPRT). It produces the high efficiency and accuracy. It also reduces the misclassified edges and boundaries. 


\section{ACKNOWLEDGEMENTS}

The authors would like to thank their colleagues for their valuable reviews and comments.

\section{REFERENCES:}

[1] A. Trémeau and P. Colantoni, (2000) "Regions adjacency graph applied to color image Segmentation," IEEE Trans. Image Process., vol. 9, no. 4, pp. 735-744, Apr.

[2] A. Delong, (2010) A. Osokin, H. Isack, and Y. Boykov, "Fast approximate energy minimization with label costs," in Proc. IEEE Conf. Comput. Vis. Pattern Recognit., San Francisco, CA, pp. 2173-2180 Jun.

[3] B. Paul, L. Zhang, and X.Wu, (2005) "Canny edge detection enhancement by scale multiplication,"IEEE. Trans. Pattern Anal. Mach. Intell., vol. 27, no. 9, pp. 1485-1490, Sept.

[4] B. Ayed and A. Mitiche, (2008) "A region merging prior for variational level set image segmentation," IEEE Trans. Image Process., vol. 17, no. 12, pp. 2301-2311, Dec.

[5] B. Marcotegui and S. Beucher, (2005) "Fast implementation of waterfall based on graphs," in Volume 30 of Computational Imaging and Vision. Dordrecht, Germany: Springer-Verlag, pp. 177-186.

[6] D. Martin, C. Fowlkes, D. Tal, and J. Malik, (2001) “A database of human segmented natural Images and its application to evaluating segmentation algorithms and measuring ecological Statistics," in Proc. ICCV, pp. 416-423.

[7] D. Comanicu and P. Meer, (2002) "Mean shift: A robust approach toward feature space analysis,"IEEE Trans. Pattern Anal. Mach. Intell., vol. 24, no. 5, pp. 603-619, May.

[8] F. Moscheni, S. Bhattacharjee, and M.Kunt, (1998) "Spatio-temporal segmentation based on region merging," IEEE Trans. Pattern Anal. Mach. Intell., vol. 20, no. 9, pp. 897-915, Sep.

[9] F. Calderero and F. Marques, (2010) "Region merging techniques using information theory Statistical measures," IEEE Trans. Image Process., vol. 19, no. 6, pp. 1567-1586, Jun.

[10] F. Lecumberry, A. Pardo, and G. Sapiro, (2010) "Simultaneous object classification and segmentation with high-order multiple shape models," IEEE Trans. Image Process., vol. 19, no. 3, pp. 625-635, Mar.

[11] H. Cheng and Y. Sun, (2000) "A hierarchical approach to color image segmentation using Homogeneity," IEEE Trans. Image Process., vol. 9, no. 12, pp. 2071-2082, Dec.

[12] J. Shi and J. Malik, (2000) "Normalized cuts and image segmentation," IEEE Trans. Pattern Anal. Mach. Intell., vol. 22, no. 8, pp. 888-905, Aug.

[13] K. Haris, S. N. Estradiadis, N. Maglaveras, and A. K. Katsaggelos, (1998) "Hybrid image Segmentation using watersheds and fast region merging," IEEE Trans. Image Process., vol. 7, no. 12, pp. 1684-1699, Dec.

[14] R. Nock and F. Nielsen, (1998) "Statistic region merging," IEEE Trans. Pattern Anal. Mach.Intell. vol. 26 , no. 11, pp. 1452-1458, Nov.

[15] S.Wang and J.M. Siskind, (2003) "Image segmentation with ratio cut," IEEE Trans. Pattern Anal Mach. Intell., vol. 25, no. 6, pp. 675-690, Jun.

[16] S. Lee and M. M. Crawford, (2005) "Unsupervised multistage image classification using Hierarchical clustering with a Bayesian similarity measure," IEEE Trans. Image Process., vol. 14, no. 3, pp. 312-320, Mar.

[17] T.Wan, N.Canagarajah, and A.Achim, (2007) "Statistical multiscale image segmentation via alpha-stable modeling," in Proc. IEEE ICIP, vol. 4, pp. IV-357-IV360.

[18] Y. Boykov and V.Kolmogorov, (2004) "An experimental comparison of min-cut/max-flow algorithms for energy minimization in vision," IEEE Trans. Pattern Anal. Mach. Intell., vol.26, no. 9, pp. 1124-1137, Sep.

[19] Luis Garcia Ugarriza, Eli Saber, Sreenath Rao Vantaram, Vincent Amuso, Mark Shaw, and RanjitBhaskar,(2009)“Automatic image segmentation by dynamic region growth and multiresolution merging", IEEE Transactions on image processing, vol.18. no.10, pp.2275 -2288, Oct.

\section{BIOGRAPHIES:}

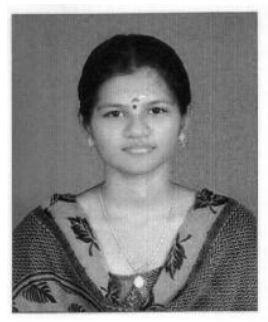

S.Muthamizhselvi received the B.E. degree in Electronics and communication engineering from Sree Sowdambika college of Engineering under Anna University, Tirunelveli, Tamil Nadu, India in 2011 and doing M.E. degree in Communication Systems from RVS College of Engineering and Technology, Coimbatore, Tamil Nadu, India. She has presented papers in the national and International conferences.

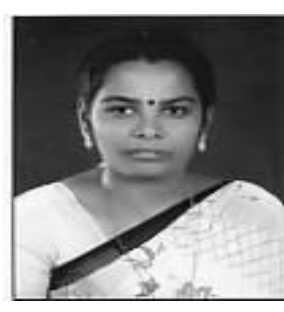

D.Jeyakumari received the B.E. degree in Electronics and communication engineering from Madurai Kamaraj University, Madurai, Tamil Nadu, India in 1997 and M.E. degree in Applied Electronics from Government College of Technology, Coimbatore, Tamil Nadu, and India in 2005. She is doing her Ph.D.

Programme in Anna University, Coimbatore. She has 16 years of teaching experience. She has published 5 papers in the reputed international journals and 20 papers in the national and international conferences. Presently working as an Associate Professor in ECE Department at RVS College Engineering and Technology, Coimbatore. She is a life member of ISTE. 


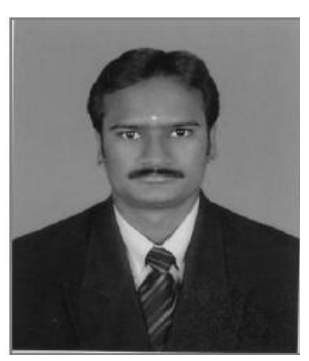

R.Kannan received the B.E. degree in Electronics and communication engineering from Dr.Sivanti Adithanar college of Engineering, Tiruchendur, Tamil Nadu, India in 2004 and M.E. degree in VLSI Design from Sona College of Technology, Salem, Tamil $\mathrm{Nadu}$, and India in 2007. He has 7 years of teaching experience. He has presented papers in the national and international conferences. Presently working as an Assistant Professor in ECE Department at RVS College Engineering and Technology, Coimbatore. 